This item was submitted to Loughborough's Research Repository by the author.

Items in Figshare are protected by copyright, with all rights reserved, unless otherwise indicated.

\title{
Classical and quantum dynamics of a particle in a narrow angle
}

PLEASE CITE THE PUBLISHED VERSION

https://doi.org/10.1134/S156035471906008X

PUBLISHER

MAIK Nauka/Interperiodica

VERSION

AM (Accepted Manuscript)

PUBLISHER STATEMENT

This paper was accepted for publication in the journal Regular and Chaotic Dynamics and the definitive published version is available at https://doi.org/10.1134/S156035471906008X

\section{LICENCE}

CC BY-NC-ND 4.0

\section{REPOSITORY RECORD}

Dobrokhotov, S, D Minenkov, Anatoly Neishtadt, and S Shlosman. 2019. "Classical and Quantum Dynamics of a Particle in a Narrow Angle". figshare. https://hdl.handle.net/2134/9939245.v1. 


\title{
Classical and quantum dynamics of a particle in a narrow angle.
}

\author{
S. Yu. Dobrokhotov, D. S. Minenkov,$\quad$ A. I. Neishtadt $† \quad$ S. B. Shlosman $\ddagger$
}

\begin{abstract}
We consider the 2D Schrödinger equation with variable potential in the narrow domain diffeomorphic to the wedge with the Dirichlet boundary condition. The corresponding classical problem is the billiard in this domain. In general, the corresponding dynamical system is not integrable. The small angle is a small parameter which allows one to make the averaging and reduce the classical dynamical system to an integrable one modulo exponential small correction. We use the quantum adiabatic approximation (operator separation of variables) to construct the asymptotic eigenfunctions (quasimodes) of the Schrödinger operator. We discuss the relation between classical averaging and constructed quasimodes. The behavior of quasimodes in the neighborhood of the cusp is studied. We also discuss the relation between Bessel and Airy functions that follows from different representations of asymptotics near the cusp.
\end{abstract}

MSC2010: 35Q40, 35J10, 35P20

Keywords: potential well, stationary Schrödinger equation, KAM-theory, operator separation of variables, semiclassical asymptotics, Airy function, Bessel function

\section{Introduction.}

Potential well problems form an important part of quantum mechanics [1]. A well could be organized by growing potential outside some domain or by suitable Dirichlet conditions at the boundary of this domain. In the first case the wave function is small outside considered domain and we speak about soft walls, in the second case it vanishes on the boundary and we speak about hard walls. The principal symbol of quantum operator can be considered as a Hamiltonian for related classical dynamical system. This system with boundary reflection condition forms a billiard problem. In cases with two and more dimensions semiclassical asymptotics for the quantum problem in a well are closely related with integrability properties of the corresponding classical billiard. To construct eigenvalues and eigenfunctions (or asymptotic eigenvalues and quasimodes) the classical problem should be integrable or nearly integrable [2].

*A.Ishlinsky Institute for Problem in Mechanics, Prospekt Vernadskogo 101-1, Moscow, 119526, Russia; Moscow Institute of Physics and Technology, 9 Institutskiy per., Dolgoprudny, Moscow Region, 141701, Russia E-mail: dobr@ipmnet.ru, minenkov.ds@gmail.com

${ }^{\dagger}$ Space Research Institute, 84/32 Profsoyuznaya Str, Moscow, 117997, Russia;

Loughborough University, Epinal Way, Loughborough Leicestershire, UK

E-mail: a.neishtadt@lboro.ac.uk

${ }^{\ddagger}$ Aix-Marseille University, CNRS, CPT, Site du Pharo - 58 bd Charles Livon - 13284 Marseille Cedex 07, France; University of Toulon, CNRS, CPT, 83041 Toulon Cedex 9, France;

Skolkovo Institute of Science and Technology, Nobel str. 3, 121205, Moscow, Russia;

Institute of the Information Transmission Problems, RAS, Bolshoy Karetny per. 19, build.1, Moscow 127051 Russia, E-mail: shlosman@gmail.com 
Integrable systems appear very seldom. One of such problems, the $n$-dimensional Weyl chamber $x_{1} \geq \ldots \geq x_{n} \geq 0$ with potential $U\left(x_{1}, \ldots, x_{n}\right)=\sum_{k=1}^{n} V\left(x_{k}\right)$, is considered in [3]. In two dimensions the Weyl chamber is the $\pi / 4$ angle. In general, the wedge with curved boundaries corresponds to a classical system that is not integrable. The presence of a potential without special symmetries also breaks the integrability. Nearly integrable cases are much more common. We consider a two-dimensional narrow angle $(\bar{x}, q) \in \mathbb{R}_{+}^{2}$ with boundaries $0 \leq q \leq d(\varepsilon \bar{x})$, where $0<\varepsilon \ll 1$ is a small parameter and positive function $d(x)>0, x>0 ; d(0)=0 ; d^{\prime}(0)>0$, and pose stationary Schrödinger equation with slowly varying potential $U(\varepsilon \bar{x}, q)$. Due to slow dependence on $\bar{x}$ the classical system becomes nearly integrable and the adiabatic perturbation theory [4] can be applied. Namely, the classical Hamiltonian can be averaged with respect to transverse variable $q$ so that its principal symbol and subprincipal symbols (up to any required order) become integrable. Averaged Hamiltonian contains effective potential $\lambda(x), x=\varepsilon \bar{x}$ with additional term proportional to $1 / d(x)^{2}$ and at the angle cusp $x \rightarrow 0, \lambda(x) \rightarrow \infty$. Adiabatic averaging (variable transform) of the classical system corresponds to a reduction in the quantum problem. Namely, one can perform operator separation of variables (see [5]) and reduce the initial problem to two one-dimensional problems. After such procedure asymptotics are defined by the reduced Schrödinger equation with potential $\lambda(x)$, which equation is the appropriate quantization of the classical averaged Hamiltonian.

Physically a narrow angle can model pinched waveguide. Waveguides are widely studied (see e.g. textbooks [6, chapter 2], where slightly perturbed waveguides are considered, and [7, chapter 7] for acoustic waveguides with varying width). In [8] operator separation of variables is applied for waveguides with varying width and reduced Schrödinger equation is studied. In this paper we are interested in the following questions: how does the reflection from the angle cusp happen and how does the cusp affect the semiclassical asymptotics. Near the cusp there is classically forbidden area that is smaller for larger energies, so the angle cusp is a focal point of a special kind. Because of fast growth of effective potential near the cusp, the momentum changes rapidly. From the classical point of view there is a special regime of motion near the turning point close to the cusp. This means that asymptotics change their structure: instead of Airy function, usually used near turning points, the Bessel function of large order could be more suitable near the cusp. Thus another interesting point is how does Airy and Bessel representation relates (see Nicholson-type formulas in $[9,10,11,12])$.

Let us pass to the mathematical formulations. We pose the Dirichlet problem for the Schrödinger equation for wavefunction $\psi(x, q)$

$$
-\frac{1}{2}\left(\frac{\partial^{2}}{\partial q^{2}}+\frac{\partial^{2}}{\partial \bar{x}^{2}}\right) \psi=(E-U(\varepsilon \bar{x}, q)) \psi,\left.\quad \psi\right|_{q=0}=0,\left.\quad \psi\right|_{q=d(\varepsilon \bar{x})}=0 .
$$

with energy $E$ and potential $U(x, q) \rightarrow \infty$, as $x \rightarrow \infty$, and study behavior of asymptotics near the angle cusp $\bar{x}=q=0$. Corresponding classical Hamiltonian is $H(\bar{x}, \xi, q, p)=\frac{1}{2}\left(p^{2}+\xi^{2}\right)+U(\bar{x}, q)$ with momenta $\xi, p$.

Introduce slow variable $x=\varepsilon \bar{x}$ and get the problem in singular perturbed setting

$$
-\frac{1}{2}\left(\frac{\partial^{2}}{\partial q^{2}}+\varepsilon^{2} \frac{\partial^{2}}{\partial x^{2}}\right) \psi=(E-U(x, q)) \psi,\left.\quad \psi\right|_{q=0}=0,\left.\quad \psi\right|_{q=d(x)}=0 .
$$

Outside some vicinity of the angle cusp this problem can be considered as a waveguide problem. Curved boundaries prevent exact separation of variables in (1.1) even without potential. Nevertheless we can apply the procedure of operator separation of variables proposed in [5] to get 1D reduced Schrödinger equation. Procedure of operator separation of variables is related to the classical adiabatic averaging: the principal symbol of reduced quantum problem is the averaged classical Hamiltonian. We construct asymptotics for the reduced Schrödinger equation by Maslov canonical operator and an appropriate coordinate transform as in [3]. Near the turning point, that 
(a)

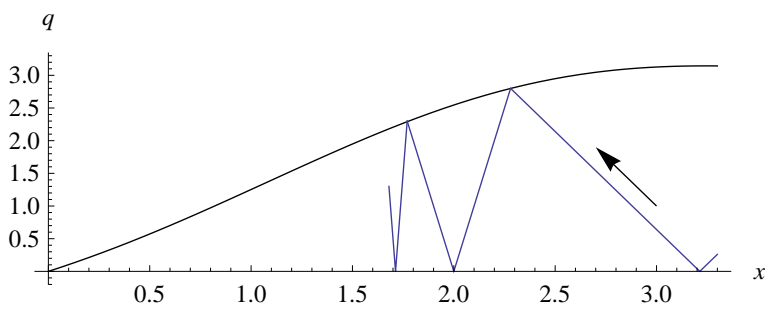

(b)

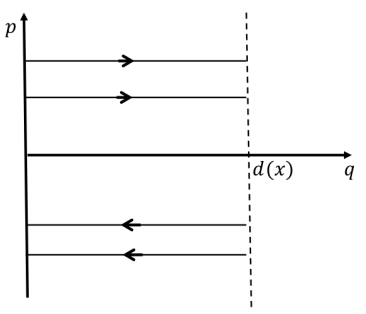

Figure 1. (a) Classical trajectories in a narrow angle.

(b) Phase portrait of motion with frozen $x$ in $(q, p)$ plane.

is close to the angle cusp, we get two representations: using Airy and Bessel functions. Their combination gives relation between these two special functions.

The paper is organized as follows. In the sec. 2 we consider classical statement and perform averaging to get integrable principal symbol of the Hamiltonian. In sec. 3 we apply operator separation of variables for the quantum problem that corresponds to transform in the classical problem from sec. 2. This allows to construct semiclassical asymptotics in sec. 4 . In sec. 5 we discuss relation between Bessel and Airy functions that follows from canonical operator asymptotics.

\section{Classical motion in a narrow angle}

\section{Original system}

Construction of asymptotics of discrete spectrum is related to integrability properties of corresponding classical Hamiltonian system. So we start with the classical case and study how adiabatic averaging leads to nearly integrable Hamiltonian.

We consider free motion of a particle of mass $m=1$ in a planar channel with ideal reflections at channel's boundaries. In Cartesian coordinates $\bar{x}, q$, boundaries of this channel have equations $q=0$ and $q=d(\varepsilon \bar{x})$, where $\varepsilon>0$ is a small parameter, and $d(\cdot)$ is a smooth function (Fig. 1 ig (a)). For motion in an angle, $d=d_{0}+d_{1} \varepsilon \bar{x}$. We denote $x=\varepsilon \bar{x}$.

Let $\xi, p$ be momenta conjugate to $\bar{x}, q$. Then motion between collisions is described by the Hamiltonian system with the Hamiltonian function

$$
E=\frac{1}{2}\left(\xi^{2}+p^{2}\right)
$$

and pairs of conjugate variables $(p, q),\left(\xi, \varepsilon^{-1} x\right)$.

\section{Hamiltonian in action-angle variables}

We use a standard approach of the adiabatic perturbation theory [4, sec. 6.4.4], but apply it for a system with collisions, like in [15]. Consider motion for frozen $x$ first. For $q, p$ we have a Hamiltonian system with one degree of freedom. The phase portrait of this system is shown in Fig. 1 (b). Let $1, \varphi$ mod $2 \pi$ be action-angle variables of this system,

$$
I=\frac{1}{2 \pi} 2|p| d=\frac{|p| d}{\pi}, \quad \varphi=\left\{\begin{array}{l}
\pi \frac{q}{d}, p \geq 0, \\
\pi\left(2-\frac{q}{d}\right), p<0 .
\end{array}\right.
$$

The generating function of the transformation $q, p \mapsto \varphi, I$ is

$$
W(q, I, x)=\left\{\begin{array}{l}
\pi I \frac{q}{d}, p \geq 0, \\
\pi I\left(2-\frac{q}{d}\right), p<0 .
\end{array}\right.
$$


The Hamiltonian in the new variables is

$$
E=\frac{1}{2} \xi^{2}+\frac{\pi^{2} I^{2}}{2 d^{2}(x)}
$$

Let us make in the original system the canonical transformation of variables $\left(q, p, \varepsilon^{-1} x, \xi\right) \mapsto$ $\left(\varphi, I, \varepsilon^{-1} x, \bar{\xi}\right)$ with the generation function

$$
\varepsilon^{-1} x \bar{\xi}+W(q, I, x)
$$

We have

$$
\xi=\bar{\xi}+\varepsilon \frac{\partial W}{\partial x}=\bar{\xi}-\varepsilon \frac{I d^{\prime}(x)}{d(x)} f(\varphi), \text { where } d^{\prime}(x)=\frac{\partial d(x)}{\partial x}, f(\varphi)=\left\{\begin{array}{l}
\varphi, \varphi \in(0, \pi), \\
\varphi-2 \pi, \varphi \in(\pi, 2 \pi) .
\end{array}\right.
$$

The Hamiltonian in the new variables is

$$
E=\frac{1}{2}\left(\bar{\xi}-\varepsilon \frac{I d^{\prime}(x)}{d(x)} f(\varphi)\right)^{2}+\frac{\pi^{2} I^{2}}{2 d^{2}(x)}=H_{0}(I, \bar{\xi}, x)+\varepsilon H_{1}(I, \varphi, \bar{\xi}, x)+\varepsilon^{2} H_{2}(I, \varphi, \bar{\xi}, x),
$$

eq_classic.

where

$$
H_{0}=\frac{1}{2} \bar{\xi}^{2}+\frac{\pi^{2} I^{2}}{2 d^{2}(x)}, H_{1}=-\bar{\xi} \frac{I d^{\prime}(x)}{d(x)} f(\varphi), H_{2}=\frac{1}{2}\left(\frac{I d^{\prime}(x)}{d(x)} f(\varphi)\right)^{2} .
$$

In the principal approximation, invariant $2 \mathrm{D}$ surfaces in $4 \mathrm{D}$ phase space are given by relations $H_{0}=h=$ const, $I=$ const, i.e

$$
p= \pm \frac{\pi I}{d(x)}, \xi= \pm \sqrt{2 h-\frac{\pi^{2} I^{2}}{d^{2}(x)}}, 0 \leq q \leq d(x) .
$$

As we will see below, averaged classical Hamiltonian $H_{0}$ is the principal symbol of corresponding reduced quantum equation and invariant manifold (2.3) relates to semiclassical asymptotics.

\section{Classical corrections}

Let us make an almost identical canonical transform of variables $\left(\varphi, I, \varepsilon^{-1} x, \bar{\xi}\right) \mapsto\left(\psi, J, \varepsilon^{-1} X, \Xi\right)$ such that the Hamiltonian function in the new variables does not contain $\psi$ in terms of the first order in $\varepsilon$. We are looking for the generating function of this transform in the form

$$
J \varphi+\varepsilon^{-1} \Xi x+\varepsilon S_{1}(J, \varphi, \Xi, x),
$$

where $S$ is $2 \pi$-periodic in $\varphi$. The old and new variables are related as follows:

$$
I=J+\varepsilon \frac{\partial S_{1}}{\partial \varphi}, \psi=\varphi+\varepsilon \frac{\partial S_{1}}{\partial J}, \bar{\xi}=\Xi+\varepsilon^{2} \frac{\partial S_{1}}{\partial x}, X=x+\varepsilon^{2} \frac{\partial S_{1}}{\partial \Xi} .
$$

Let $\mathcal{H}_{1}(J, \Xi, X)$ be the term of order $\varepsilon$ in the Hamiltonian for the new variables. Then

$$
\frac{\partial H_{0}(I, \xi, x)}{\partial I} \frac{\partial S_{1}(I, \varphi, \xi, x)}{\partial \varphi}+H_{1}(I, \varphi, \xi, x)=\mathcal{H}_{1}(I, \xi, x) .
$$

As $S_{1}$ is $2 \pi$-periodic in $\varphi$, we get

$$
\begin{aligned}
\mathcal{H}_{1}(I, \xi, x) & =\frac{1}{2 \pi} \int_{0}^{2 \pi} H_{1}(I, \varphi, \xi, x) d \varphi \\
S_{1}(I, \varphi, \xi, x) & =-\frac{1}{\partial H_{0}(I, \xi, x) / \partial I} \int_{0}^{\varphi}\left(H_{1}(I, \theta, \xi, x)-\mathcal{H}_{1}(I, \xi, x)\right) d \theta+S_{1}^{0}(I, \xi, x),
\end{aligned}
$$


where $S_{1}^{0}(I, \xi, x)$ is an arbitrary function. In our case $\mathcal{H}_{1} \equiv 0, H_{1}=-\xi \frac{I d^{\prime}(x)}{d(x)} f(\varphi), H_{0}=\frac{1}{2} \xi^{2}+$ $\frac{\pi^{2} I^{2}}{2 d^{2}(x)}$. Thus we have

$$
\frac{\partial S_{1}(\varphi, I, \xi, x)}{\partial \varphi}=\frac{\xi d d^{\prime}}{\pi^{2}} f(\varphi)
$$

Hence

$$
I=J+\varepsilon \frac{\partial S}{\partial \varphi}=J+\frac{\xi d d^{\prime}}{\pi^{2}} f(\varphi)=J+\varepsilon \frac{\xi d^{\prime}}{\pi} q \operatorname{sgn}(p) .
$$

In the first approximation, invariant $2 \mathrm{D}$ surfaces in $4 \mathrm{D}$ phase space are given by relations $H_{0}(J, \bar{\xi}, x)=$ $h=$ const, $J=$ const, i.e

$$
p= \pm \frac{\pi}{d(x)}\left(J+\varepsilon \frac{\xi d^{\prime}}{\pi} q \operatorname{sgn}(p)\right), \quad \xi= \pm \sqrt{2 h-\frac{\pi^{2} J^{2}}{d^{2}(x)}}-\varepsilon \frac{\pi J d^{\prime}}{d^{2}} q \operatorname{sgn}(p) .
$$

Such procedure can be continued to make averaged Hamiltonian as precise as we want.

In what follows we discuss the role of described classical objects in quantum problem and the relation between classical and quantum problems.

\section{Operator separation of variables}

Here we perform operator separation of variables and study the relation between averaged equation and averaged classical Hamiltonian.

Consider Schrödinger equation (1.1). Standard adiabatic approach is based on Born and Oppenheimer works [16] and was adopted for fast oscillating solutions like WKB in [17]. The general method of operator separation of variables was formulated in [5] and it uses ideas of Peierls substitution $[18,19]$. We will follow it in the way described in [8] (see also [20, 21]).

Here and below we consider the following quantization rule

$$
\hat{f}=f(\hat{\xi}, \stackrel{2}{x}), \quad \hat{\xi}=-i \varepsilon \frac{\partial}{\partial \xi}
$$

Denote $\mathcal{H}\left(\xi, \frac{\partial}{\partial q}, x, q\right)=\frac{1}{2}\left(\xi^{2}-\frac{\partial^{2}}{\partial q^{2}}\right)+U(x, q)$, so that $\left(\frac{\text { eq_Sch } 1 \text { 2D }}{1.1}\right.$ can be written as $\hat{\mathcal{H}} \psi(x, q)=E \psi(x, q)$. We are looking for solution in the form $\psi(x, q)=\hat{w} \varphi(x), \hat{w}=w(\hat{\xi}, x, q)$ and we want to get equation $\hat{H} \varphi(x)=E \varphi(x), \hat{H}=H(\hat{\xi}, x, q)$. This gives

$$
\hat{\mathcal{H}} \hat{w} \varphi=\hat{w} \hat{H} \varphi .
$$

It is sufficient to have operator equality $\hat{\mathcal{H}} \hat{w}=\hat{w} \hat{H}$, and corresponding equations for the symbols $H=H_{0}+\varepsilon H_{1}+O\left(\varepsilon^{2}\right), w=w_{0}+\varepsilon w_{1}+O(\varepsilon)$ are

$$
\begin{aligned}
& \left(\mathcal{H}\left(\xi, \frac{\partial}{\partial q}, x, q\right)-H_{0}(\xi, x)\right) w_{0}(\xi, x, q)=0 \\
& \left(\mathcal{H}\left(\xi, \frac{\partial}{\partial q}, x, q\right)-H_{0}(\xi, x)\right) w_{1}(\xi, x, q)-i \mathcal{H}_{\xi} w_{0 x}=w_{0} H_{1}-i w_{0 \xi} H_{0 x}
\end{aligned}
$$

Symbols $w_{n}$ also satisfy the Dirichlet boundary conditions $\left.w_{n}\right|_{q=0}=0,\left.w_{n}\right|_{q=d(x)}=0$.

Normalization condition $\|\psi(x, q)\|_{x, q}=1$ leads to equality $\|\phi\|_{x}=1$ and operator equation $\hat{w}^{*} \hat{w}=1$ that gives chain of equations for symbols

$$
\left\|w_{0}\right\|_{q}=1, \quad 2\left\langle w_{0}, w_{1}\right\rangle_{q}-i\left\langle w_{0 \xi}, w_{0 x}\right\rangle=0 .
$$


Here the scalar product is $\left\langle f(q){ }_{\text {H }} q(q)\right\rangle_{q}=\int_{0}^{d(x)} f(q) g(q) d q$.

From the first equation (3.1) we found $H_{0}(\xi, x)=\frac{1}{2} \xi^{2}+\lambda(x)$, where $\lambda(x)$ and $w_{0}=w_{0}(x, q)$ are eigenvalue and eigenfunction of the Dirichlet problem with respect to $q$ :

$$
-\frac{1}{2} \frac{\partial^{2}}{\partial q^{2}} w_{0}+U(x, q) w_{0}=\lambda(x) w_{0},\left.\quad w_{0}\right|_{q=0}=\left.w_{0}\right|_{q=d(x)}=0,
$$

while $x$ and $\xi$ are "frozen" ( $x$ and $\xi$ are considered as parameters).

To find correction $H_{1}$ we take scalar product $\left\langle w_{0}, \cdot\right\rangle_{q}$ of $w_{0}$ with the second equation $(3.2)$ :

$$
H_{1}=-i \xi\left\langle w_{0}, w_{0 x}\right\rangle_{q}=-i \xi \frac{1}{2}\left(\frac{\partial}{\partial x}\left\langle w_{0}, w_{0}\right\rangle_{q}+d^{\prime}(x) w_{0}^{2}(x, d(x))\right)=0 .
$$

Thus correction $w_{1}(\xi, x, q)$ is a solution of inhomogeneous equation

$$
-\frac{1}{2} \frac{\partial^{2}}{\partial q^{2}} w_{1}+U(x, q) w_{1}-\lambda(x) w_{1}=i \xi w_{0 x}
$$

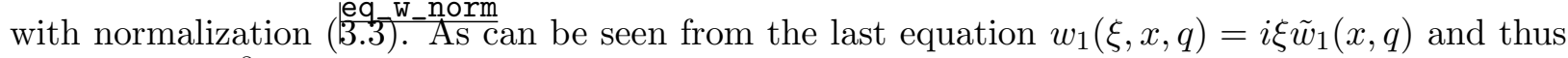
$\hat{w}_{1}=\tilde{w}_{1}(x, q) \frac{\partial}{\partial x}$ is a differential operator.

Remark 3.1. To construct the leading term of formal asymptotics to (1.1) it is enough to find $w_{0}$ and $H_{0}, H_{1}$. We only need to state the existence of solution $w_{1}$ to prove the convergence of asymptotic procedure.

Asymptotics of $\varphi(x)$ are found from the reduced Schrödinger equation

$$
\hat{H}_{0} \varphi=-\varepsilon^{2} \frac{1}{2} \frac{\partial^{2}}{\partial x^{2}} \varphi+(\lambda(x)-E) \varphi=O\left(\varepsilon^{2}\right),
$$

eq_Sch_1D

where effective potential is $\lambda(x) \sim 1 / d(x) \rightarrow \infty$ as $x \rightarrow 0$ and if $U(x, q) \rightarrow \infty$ as $x \rightarrow \infty$ then also $\lambda(x) \rightarrow \infty$. For energy $E>\min \lambda(x)$ there are two turning points $x_{-}<x_{+}: E=\lambda\left(x_{ \pm}\right)$. In the segment $x \in\left(x_{-}, x_{+}\right)$the solution oscillates. Intervals $x<x_{-}$and $x>x_{+}$are classically forbidden and solution is exponentially small there.

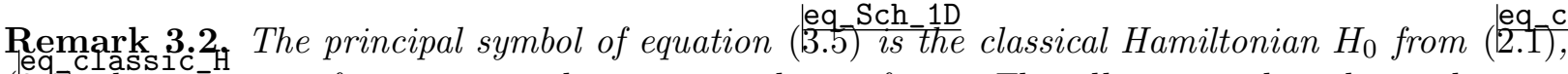
$(2.2)$ that appears after corresponding canonical transform. This illustrates the relation between classical dynamics and semi-classical asymptotics of quantum problem.

Remark 3.3. One can consider $n$-dimensional cone $(0, \infty)_{r} \times \Sigma_{q}(\varepsilon r), \Sigma_{q}(\rho) \subset \mathbb{R}^{n-1}$, where $\left(\Sigma_{q}(\rho), h(\rho)\right)$ is a family of compact $(n-1)$-dimensional Riemannian manifolds. If corresponding eigenvalues and eigenfunctions $\lambda_{\nu}(\rho), w_{\nu}(\rho)$ are smooth with respect to parameter $\rho$ then the presented procedure of operator separation of variables can be naturally generalized, and one can get effective one-dimensional Schrödinger equation for $\psi^{\nu}(x)$. Such multi-dimensional procedure is considered in [8].

\section{Example 1. Operator separation of variables for small potential}

Consider the angle with curved boundary $q=d(x)$. To have explicit formulas we assume that potential $U(x, q)=v_{0}(x)+\varepsilon v_{1}(x, q)+O\left(\varepsilon^{2}\right)$ is adiabatic with respect to $q$. Operator $-\frac{1}{2} \frac{\partial^{2}}{\partial q^{2}}$ with Dirichlet conditions has the following eigenvalues and eigenfunctions

$$
\lambda^{\nu}(x)=\frac{1}{2} \frac{\pi^{2} \nu^{2}}{d(x)^{2}}, \quad w_{0}^{\nu}(x, q)=\frac{\sqrt{2}}{\sqrt{d(x)}} \sin \left(\frac{\pi \nu}{d(x)} q\right) .
$$

Symbol of the reduced equation is

$$
\begin{aligned}
& H(\xi, x, \varepsilon)=H_{0}(\xi, x)+\varepsilon H_{1}(\xi, x)+O\left(\varepsilon^{2}\right), \\
& H_{0}(\xi, x)=\frac{\xi^{2}}{2}+v_{0}(x)+\lambda^{\nu}(x), \quad H_{1}(x)=\left\langle v_{1}(x, q), w_{0}(x, q)^{2}\right\rangle_{q} .
\end{aligned}
$$


(a)

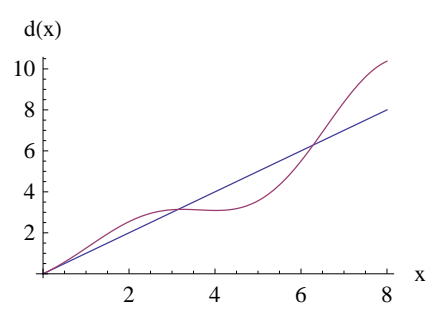

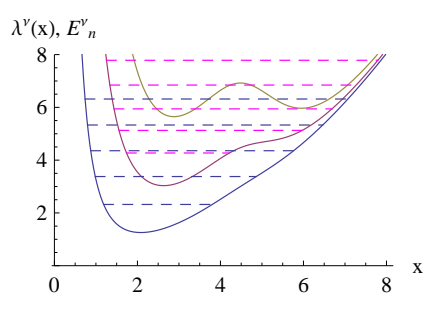

(c) -4

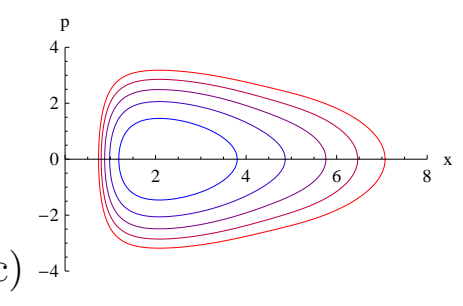

Figure 2. (a) Boundary function $q=d(x)$ (magenta) and $q=x$ (blue).

(b) Effective potential $\lambda^{\nu}(x)$ for $\nu=1,2,3$ (blue, magenta, yellow) and energy levels $E_{n}^{\nu}$ for $\nu=1,2, n=$ $10,20,30,40,50$ (blue and magenta dashed).

(c) Lagrangian curve $H(X, P)=E$ for $\nu=1$ and $n=10,20,30,40,50$ (from blue to red).

Due to additional potential $\lambda^{\nu}(x)=\frac{1}{2} \frac{\pi^{2} \nu^{2}}{d(x)^{2}} \rightarrow \infty$ as $x \rightarrow 0$ the region near the angle cusp is classically forbidden and the solution vanishes: $\psi^{\nu}(0)=0$. Let us set $v_{0}(0)=0$, then classically forbidden region $x<x_{-}(E)$ is defined by $E=\lambda^{1}\left(x_{-}\right)=\frac{\pi^{2}}{2 d\left(x_{-}\right)^{2}}+O(x)+O(\varepsilon)$, which gives $x_{-}(E) \sim E^{-1 / 2}$. Potential $\lambda^{\nu}\left(x_{1}\right)$ and Lagrangian manifolds for different modes $\nu$ and energies $E$ are illustrated by Fig. $\stackrel{F}{2}$.

\section{Asymptotics of reduced Schrödinger equation}

\section{Quantization conditions}

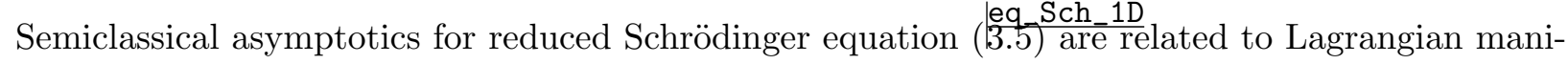
fold $\Lambda(E)$ that is defined by Hamiltonian trajectories for energy $E$ :

$$
H=\frac{\xi^{2}}{2}+\lambda(x)=E .
$$

Lagrangian manifold is a cycle. In the turning points $x=x_{ \pm}: \lambda\left(x_{ \pm}\right)=E$ Jacobian $J=\partial \xi / \partial x$ has simple roots $J\left(x_{ \pm}\right)=0, J^{\prime}\left(x_{ \pm}\right) \neq 0$ and the increment of Maslov index over the cycle is equal to $\delta m=2$, thus quantization conditions for $E=E_{n}$ are standard Bohr-Sommerfeld conditions

$$
\int_{x_{-}}^{x_{+}} \sqrt{2\left(E_{n}-\lambda(x)\right)} d x=\pi h\left(n-\frac{\delta m}{4}\right)=\pi h\left(n-\frac{1}{2}\right), \quad n=1,2, \ldots
$$

\section{Maslov canonical operator and Airy asymptotics near turning points}

Despite (3.5) is one-dimensional, we construct asymptotics using Maslov canonical operator to show how it gives Airy integral near turning points $x_{ \pm}: \lambda\left(x_{ \pm}\right)=E$. As in [3], we use canonical transform $x=X(y), \xi=\Xi(\chi, y)=\chi / X^{\prime}(y)$ (denote its inverse by $y=Y(x)$ ) with the property

$$
\frac{1}{2} R_{A}(x) Y^{\prime}(x)^{2}=1, \quad R_{A}(x)=\frac{Y(x)}{\lambda(x)-E} .
$$

Such transform changes the Hamiltonian (the principal symbol of reduced equation (3.5)) as follows:

$$
H(\Xi(\chi, y), X(y))-E=h(\chi, y, E) \equiv R_{A}^{-1}\left(\chi^{2}+y-E\right) .
$$

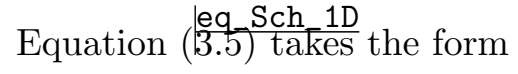

$$
-\varepsilon^{2} \frac{\partial^{2}}{\partial y^{2}} \varphi+y \varphi-\varepsilon g(y) \varepsilon \frac{\partial}{\partial y} \varphi=0, \quad g(y)=\frac{1}{\sqrt{2}} \sqrt{\left|R_{A}(X(y))\right|} Y^{\prime \prime}(X(y)) .
$$


The transform $Y(x)$ is defined by formula:

$$
Y_{ \pm}(x)= \pm \operatorname{sgn}\left(x-x_{ \pm}\right)\left(\frac{3}{2} \int_{x_{ \pm}}^{x} \sqrt{|2(\lambda(x)-E)|}\right)^{2 / 3} .
$$

This substitution is smooth; Jacobian $Y_{+}^{\prime}$ does not vanish near $x_{+}$(on any segment $\left[b_{1}, b_{2}\right] \subset$ $\left.\left(x_{-}, \infty\right)\right)$ and $Y_{-}^{\prime}$ does not vanish near $x_{-}$(on segments $\left[b_{1}, b_{2}\right] \subset\left(0, x_{+}\right)$).

New Hamiltonian $h(\chi, y, E)=0$ defines the same Lagrangian curve $\Lambda(E)$ (see Fig. Fig_u_eff $H(\xi, x)=E$ and the Hamiltonian system has the form $\frac{\partial}{\partial t} y(t)=R_{A}^{-1} \chi(t), \frac{\partial}{\partial t} \chi(t)=R_{A}^{-1}$. We change time $t \rightarrow \tau: \frac{\partial \tau}{\partial t}=R_{A}^{-1}$ to have

$$
\frac{\partial}{\partial \tau} y(\tau)=2 \chi, \quad \frac{\partial}{\partial \tau} \chi(\tau)=-1 \quad \Rightarrow \quad \chi(\tau)=-\tau, \quad y(\tau)=-\tau^{2} .
$$

Here we set integration constants to zero to have turning point $x=x_{+}$at zero time $y(0)=0$.

We introduce Jacobians $J=\frac{\partial y}{\partial \tau}= \pm \sqrt{-y}, \tilde{J}=\frac{\partial \chi}{\partial \tau}=-1$. Turning point $y\left(x_{ \pm}\right)=0$ is a focal point: $J=0$ and inverse function $\tau=\tau(y)$ can't be found. Thus we need to write phase in singular representation

$$
\tilde{S}(\chi)=-\int_{0}^{\chi} y d \chi=\chi^{3} / 3
$$

Canonical operator then is simply

$$
\begin{aligned}
K_{\Lambda(E, \tau)}^{\varepsilon}[c](y) & =\frac{c e^{i \frac{\pi}{4}}}{\sqrt{2 \pi \varepsilon}} \int_{\mathbb{R}}|\tilde{J}(\chi)|^{-1 / 2} e^{\frac{i}{\varepsilon}(\tilde{S}(\chi)+y \chi)} d \chi \\
& =\frac{c e^{i \frac{\pi}{4}}}{\sqrt{2 \pi \varepsilon}} \int_{\mathbb{R}} e^{\frac{i}{\varepsilon}\left(\frac{1}{3} \chi^{3}+y \chi\right)} d \chi=c e^{i \frac{\pi}{4}} \frac{\sqrt{2 \pi}}{\varepsilon^{1 / 6}} \operatorname{Ai}\left(\frac{y}{\varepsilon^{2 / 3}}\right) .
\end{aligned}
$$

To make the inverse transform we use the general property of canonical operator $[17,19]$ :

$$
K_{\Lambda(E, t)}^{\varepsilon}[A(t)](x)=\sqrt{\left|\frac{\partial Y}{\partial x}\right|} K_{\Lambda(E, \tau)}^{\varepsilon}\left[\sqrt{\left|\frac{\partial t}{\partial \tau}\right|} A(\tau)\right](Y(x)) .
$$

prop_CO

Finally asymptotics to $(\stackrel{\text { eq_Sch_1D }}{3.5})$ near $x_{ \pm}$is [3]

$$
\varphi(x)=c e^{i \frac{\pi}{4}} \frac{\sqrt{2 \pi}}{\varepsilon^{1 / 6}}\left(2 R_{A}^{ \pm}(x)\right)^{1 / 4} \operatorname{Ai}\left(\frac{Y_{ \pm}(x)}{\varepsilon^{2 / 3}}\right)(1+O(\varepsilon)),
$$

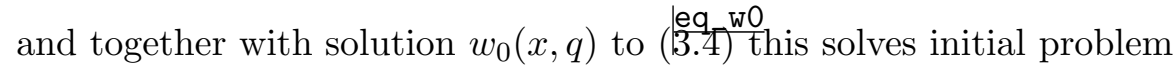

$$
\psi(x, q)=w_{0}(x, q) \varphi(x)+O(\varepsilon) .
$$

\section{Bessel asymptotics near the angle cusp}

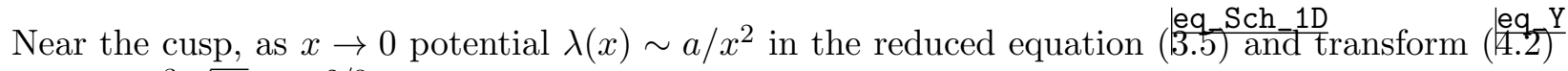
$Y(x) \sim\left(\frac{3}{2} \sqrt{2 a} \ln x\right)^{2 / 3}$ are not bounded. For large energies (focal) turning point $x_{-}$approaches the cusp and formally considerations of previous section fails. Near the cusp it is more natural to use Bessel-type asymptotics.

Assume $d^{\prime}(0)=\gamma>0$ so that effective potential $\lambda(x)=\frac{a}{x^{2}}(1+O(x)), a=\frac{\pi^{2} \nu^{2}}{2 \gamma^{2}}$ as $x \rightarrow 0$. Consider canonical transforms in the phase space $x=\tilde{X}(z), \xi=\tilde{\Xi}(\zeta, z)=\zeta / \tilde{X}^{\prime}(z)$ (with inverse $z=Z(x))$, so that the Hamiltonian changes in the following way:

$$
H(\tilde{\Xi}(\zeta, z), \tilde{X}(z))-E=\tilde{h}(\zeta, z) \equiv R_{B}^{-1}\left(\frac{1}{2} \zeta^{2}-\left(E-\frac{a}{z^{2}}\right)\right), \quad R_{B}(x)=\frac{E-a / Z(x)^{2}}{E-\lambda(x)} .
$$


(a)

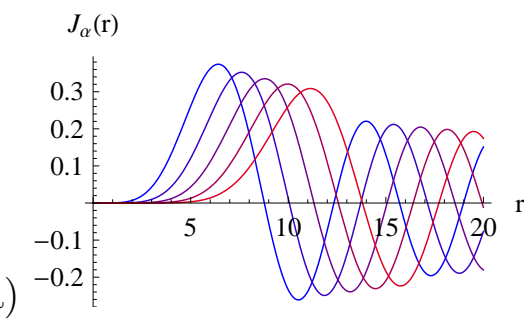

(b) -0.2

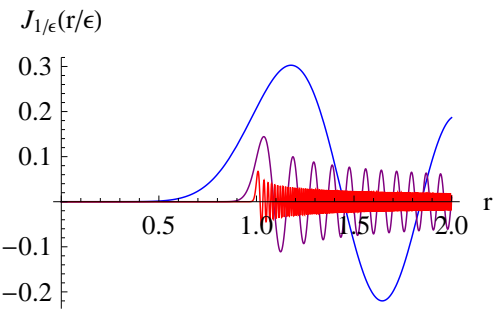

Figure 3. (a) Bessel function $J_{\alpha}(r)$ for $\alpha=5,6.1,7.2,8.3,9.4$ (from blue to red). (b) $J_{1 / \varepsilon}(r / \varepsilon)$ for $\varepsilon=0.1,0.01,0.001$ (from blue to red).

This transform satisfies

$$
R_{B}\left(Z^{\prime}(x)\right)^{2}=1 \quad \Leftrightarrow \quad \int_{z_{0}}^{Z(x)} \sqrt{\left|E-a / z^{2}\right|} d z=\int_{x_{-}}^{x} \sqrt{|E-\lambda(x)|} d x
$$

where $z_{0}=Z\left(x_{-}\right)=\sqrt{a / E}$. This transform is smooth and bounded near the cusp $Z(x) \sim x+O\left(x^{2}\right)$ as $x \rightarrow 0 ; Z^{\prime}$ does not vanish on intervals $\left(0, b_{1}\right] \subset\left(0, x_{+}\right)$. Equation (3.5) becomes

$$
-\frac{\varepsilon^{2}}{2} \frac{\partial^{2}}{\partial z^{2}} \varphi-\left(E-\frac{a}{z^{2}}\right) \varphi-\frac{\varepsilon}{2} f(z) \varepsilon \frac{\partial}{\partial z} \varphi=0, \quad f(z)=\sqrt{\left|R_{B}(z)\right|} Z^{\prime \prime}(\tilde{X}(z)) .
$$

If we omit small term $\frac{\varepsilon}{2} f(z) \varepsilon \frac{\partial}{\partial z} \varphi$ its solution is (see [22, sec. 1.4])

$$
\varphi_{0}(x)=\left.c_{0} \sqrt{z} J_{\alpha_{1}}\left(\frac{\sqrt{2 E}}{\varepsilon} z\right)\right|_{z=Z(x)}, \quad \alpha_{1}=\sqrt{\frac{1}{4}+\frac{2 a}{\varepsilon^{2}}}=\frac{\sqrt{2 a}}{\varepsilon}+O(\varepsilon) .
$$

Behavior of Bessel function of large or Fig_Bessel_large_alpha positive zero $r=\varepsilon \mu_{1}>0$ of function $J_{b / \varepsilon}(r / \varepsilon)=0$ is $\mu_{1}=b \varepsilon^{-1}+c b^{1 / 3} \varepsilon^{-1 / 3}+O\left(\varepsilon^{1 / 3}\right), c \approx 1.86$ so that $r=b+O\left(\varepsilon^{2 / 3}\right)$ as $\varepsilon \rightarrow \infty$ (see [11, sec. 15 - 82]).

New Hamiltonian $\tilde{h}=0$ as $H=E$ defines the same Lagrangian curve $\Lambda(E)$ that can be defined as $z=\mathcal{Z}(\zeta)=\sqrt{2 a} / \sqrt{2 E-\zeta^{2}}$. We change time for Hamiltonian system $t \rightarrow \tau, d \tau / d t=R_{B}^{-1}$ and get $\frac{\partial}{\partial \tau} z=\zeta, \frac{\partial}{\partial \tau} \zeta=-\frac{2 a}{z^{3}}$. Jacobians are $J(z)=\frac{\partial z}{\partial \tau}= \pm \sqrt{2 E-2 a / z^{2}}, \tilde{J}(\zeta)=\frac{\partial \zeta}{\partial \tau}=$ $-\left(2 E-\zeta^{2}\right)^{3 / 2} / \sqrt{2 a}<0$. Phase in focal map is

$$
\tilde{S}(\zeta)=-\int_{0}^{\zeta} z(\zeta) d \zeta=-\sqrt{2 a} \arcsin \left(\frac{\zeta}{\sqrt{2 E}}\right)
$$

Canonical operator gives integral representation of Bessel function of order $\alpha=\sqrt{2 a} / \varepsilon$

$$
\begin{aligned}
K_{\Lambda(E, \tau)}^{\varepsilon}[c](z) & =\frac{c e^{i \frac{\pi}{4}}}{\sqrt{2 \pi \varepsilon}} \int_{\mathbb{R}}|\tilde{J}(\zeta)|^{-1 / 2} e^{\frac{i}{\varepsilon}(\tilde{S}(\zeta)+z \zeta)} d \zeta \\
& =\frac{c e^{i \frac{\pi}{4}}}{\sqrt{2 \pi \varepsilon}} \int_{\mathbb{R}} \sqrt{\mathcal{Z}(\zeta(\theta))} e^{\frac{i}{\varepsilon}(\sqrt{2 E} z \sin \theta-\sqrt{2 a} \theta)} d \theta=c e^{i \frac{\pi}{4} \frac{\sqrt{2 \pi}}{\sqrt{\varepsilon}} \sqrt{z} J_{\alpha}\left(\frac{\sqrt{2 E}}{\varepsilon} z\right) .} .
\end{aligned}
$$

Here we make substitution $\zeta=\zeta(\theta)=\sqrt{2 E} \sin \theta$, and use the well-known commutation property

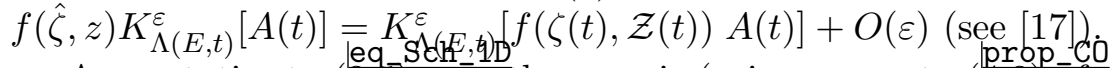

Asymptotics to (3.5) near the cusp is (using property (14.3) of canonical operator)

$$
\varphi(x)=R_{B}(x)^{1 / 4} K_{\Lambda(E, \tau)}^{\varepsilon}[c](Z(x))=c e^{i \frac{\pi}{4}} \frac{\sqrt{2 \pi}}{\sqrt{\varepsilon}} \sqrt{Z^{\prime}(x)} \sqrt{Z(x)} J_{\alpha}\left(\frac{\sqrt{2 E}}{\varepsilon} Z(x)\right)(1+O(\varepsilon)) .
$$

as_Bessel 


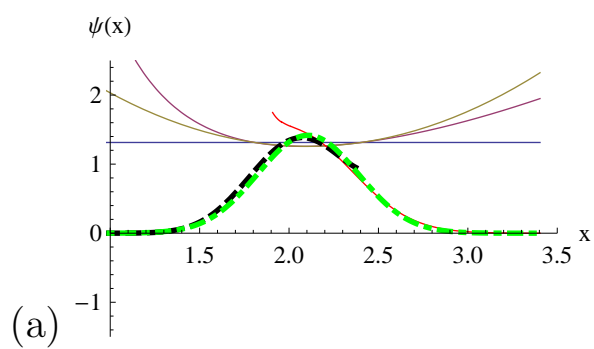

(b)
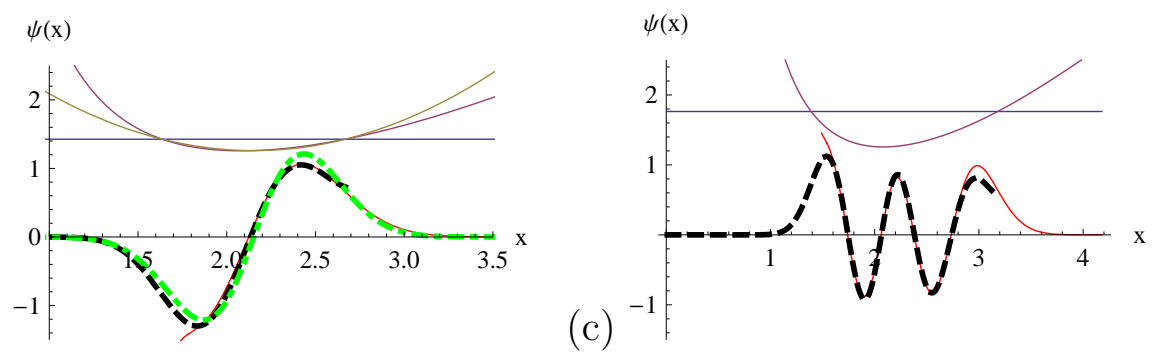

Figure 4. Asymptotics for effective Schrödinger equation for $E=E_{n}$ (a), (b) and (c): $n=1,2,5$. Asymptotics with Bessel (black dashed), with Airy (red) and harmonic oscillator (green dotdashed), ig_as energy level $E_{n}$ (blue horizontal line), potential $\lambda^{\nu}$ (magenta) and its approximation $\lambda_{\text {appr }}^{\nu}$ (yellow).

(a)

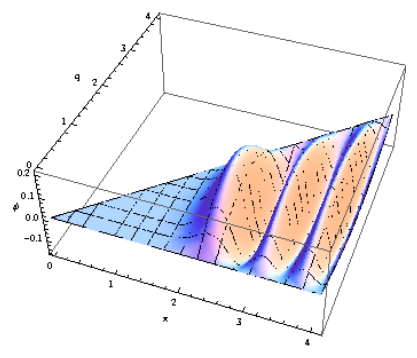

(b)

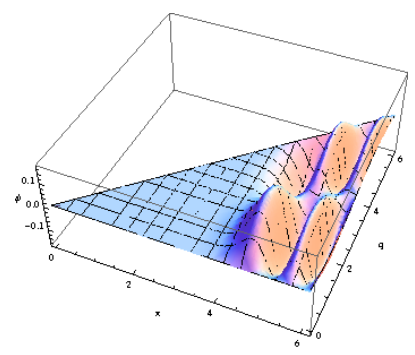

(c)

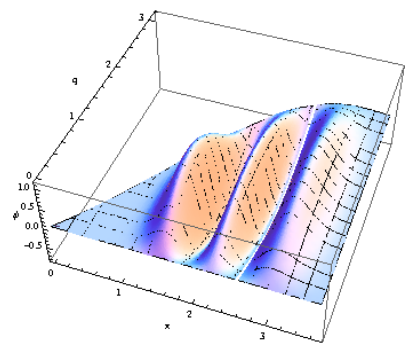

Figure 5. (a), (b): exact solution for $U_{\mathrm{sec}} 0_{\grave{a s}} d(x)=x \arctan (\varepsilon \theta) / \varepsilon, \varepsilon=0.1, \theta=1, \nu=1$ and $\nu=2$. (c) Asymptotics for Example 3 in sec. $4: 0 \stackrel{0}{=}=x^{2} / 8, d(x)=x(1+0.3 \sin x), \varepsilon=0.1, \nu=1, n=5$.

Remark 4.1. Formulas $\left(\frac{a S_{\text {Airy }} \text { A.4 }}{4.4}\right.$ and $\left(\frac{\text { as Bessel }}{4.7}\right)$ can both be used for asymptotics near the cusp. Which one to choose is the question of convenience. Coordinate transform (eq.2) is easier to compute. From the other hand, it is unbounded: $Y(x) \rightarrow \infty$ as $x \rightarrow 0$ while $Z(x)=O(1)$ and thus Bessel representation seems more natural near the cusp.

\section{Example 2. Semiclassical asymptotics}

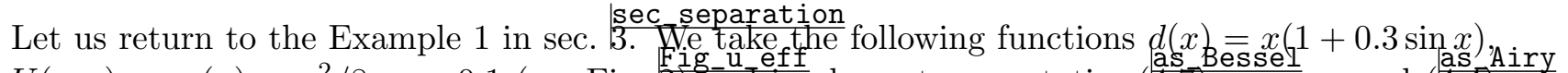

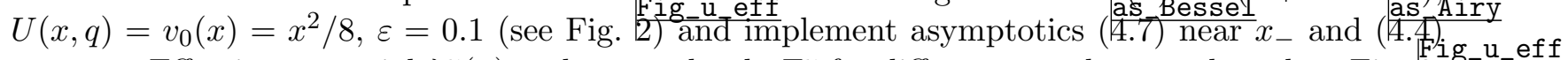
near $x_{+}$. Effective potential $\lambda^{\nu}(x)$ and energy levels $E_{n}^{\nu}$ for different $\nu$ and $n$ are plotted on Fig. 2 . We consider $\nu=1$ and energy $E_{n}$ with $n=1,2,5$.

Asymptotics of effective Schrödinger equation are presented on Fig. Fig. Tt is illustrates that asymptotics with "varying" arguments $Y(x), Z(x)$ have wide applicability region. Formulas near $x_{-}$are valid in $\left(0, x_{+}-\sqrt{\varepsilon}\right)$ and formulas near $x_{+}-$in $\left(x_{-}+\sqrt{\varepsilon}, \infty\right)$. Asymptotics provide good matching in the wide region $\left(x_{-}+\sqrt{\varepsilon}, x_{+}-\sqrt{\varepsilon}\right)$.

For small quantum numbers $n=1,2$ we also compare these asymptotics with harmonic oscillator solutions for quadratic approximation $\lambda_{a p p r}^{\nu}$ of effective potential $\lambda^{\nu}: \lambda_{a p p r}^{\nu}(x)=\lambda^{\nu}\left(x_{0}\right)+a(x-$ $\left.x_{0}\right)^{2} / 2, x_{0}=\left(x_{-}+x_{+}\right) / 2, a=2\left(E_{n}-\lambda^{\nu}\left(x_{0}\right)\right) /\left(x_{+}-x_{0}\right)^{2}$. Comparison with harmonic oscillator approximation for $n=1,2$ shows that obtained asymptotics can be applied as well for small quantum numbers. Illustration of asymptotics in angle with curved boundaries and its comparison with exact solution for a straight angle and without potential can be found on Fig. 5 .

Asymptotics $\psi(x)$ are plotted on Fig. (5) and is compared with exact solution for angle with straight boundaries and with zero potential. 

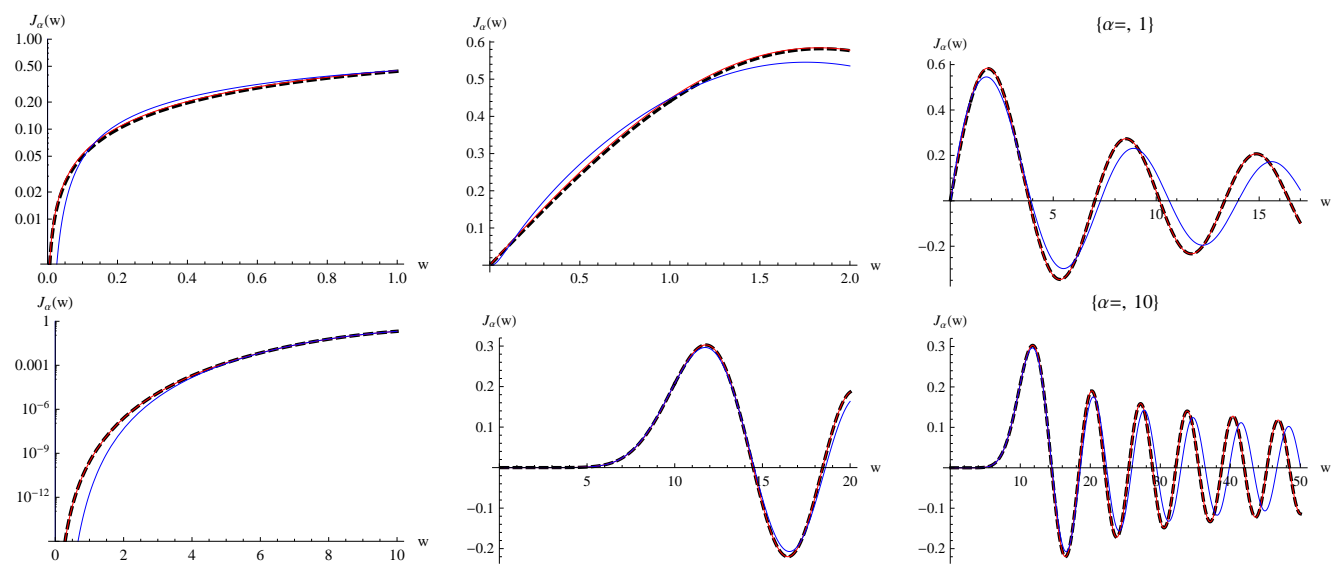

Figure 6. Comparison of approximations of Bessel function $J_{0}\left(\psi_{i}\right)$ (black dashed line) with Airy function by Nicholson-like formula (5.3) (blue) and formula (5.2) provided by canonical operator asymptotics.

The first three figures are plot for $\alpha=1$ (for three different ranges) and the last three - for $\alpha=10$.

\section{Relation between Airy and Bessel}

Here we consider angle with straight boundaries $q=0, q=d(x)=\gamma x$ and use asymptotics to study relation Airy and Bessel functions. The angle with straight boundaries is a simple cone and there are a lot of studies for Laplacians of cones (see e.g. recent results [13], that are based on [14], and bibliography within).

If potential $U(x)=0$ then the reduced equation ( 2 eq.5 Sch_1D 1 has potential $\lambda(x)=a / x^{2}, a=\pi^{2} \nu^{2} / 2 \gamma^{2}$. We set $a=1 / 2, E=1 / 2$ (this can be done by using the normalization $\varepsilon=\tilde{\varepsilon} \sqrt{2 a}, x=\tilde{x} \sqrt{a / E}$ ) and consider equation

$$
-\varepsilon^{2} \varphi^{\prime \prime}+\frac{1}{x^{2}} \varphi=\varphi, \quad \varphi=c \sqrt{x} J_{\alpha_{1}}\left(\frac{x}{\varepsilon}\right), \quad \alpha_{1}=\frac{1}{\varepsilon} \sqrt{1+\frac{\varepsilon^{2}}{4}} .
$$

For considered potential Lagrangian manifold $\Lambda_{0}$ is defined by $\xi^{2}=1-\frac{1}{x^{2}}$ and there is just one turning point is $x_{0}=1$. Coordinate transform (4.6) in this case becomes trivial $Z(x)=x$, transform $(4.2)$ is defined for $x \in \mathbb{R}_{+}$and can be explicitly integrated:

$$
Y(x)=\left\{\begin{array}{cl}
-\left(\frac{3}{2}\right)^{2 / 3}\left(\sqrt{x^{2}-1}-\operatorname{arcsec}(x)\right)^{2 / 3}, & x \geq 1, \\
\left(\frac{3}{2}\right)^{2 / 3}\left(-\ln x+\ln \left(1+\sqrt{1-x^{2}}\right)-\sqrt{1-x^{2}}\right)^{2 / 3}, & x<1 .
\end{array}\right.
$$

Here $\sec y \equiv 1 / \cos y=x$, and $y=\operatorname{arcsec} x$ is its inverse. For $x>1: \operatorname{arcsec} x \in[0, \pi / 2]$ as $\pi_{\text {Beselas_Airy }}$

Denote $\alpha=\varepsilon^{-1}$ and $w=x \varepsilon^{-1}$. Combining canonical operator asymptotics (4.7) and (4.4), we get relation between Airy and Bessel functions

$$
J_{\alpha}(w) \approx \frac{2^{1 / 4}}{\alpha^{1 / 3}} \frac{1}{\sqrt{w / \alpha}}\left(\frac{2 Y\left(\frac{w}{\alpha}\right)}{\alpha^{2} / w^{2}-1}\right)^{1 / 4} \operatorname{Ai}\left(\alpha^{2 / 3} Y\left(\frac{w}{\alpha}\right)\right)\left(1+O\left(\alpha^{-1}\right)\right) .
$$

as_BesselA

It includes complicated argument $\left(\begin{array}{l}\text { (5. } \\ 5.1\end{array}\right)$ that makes difference from the well-known Nicholson-type formulas

$$
J_{\alpha}(x) \sim\left(\frac{x}{2}\right)^{-\frac{1}{3}} \operatorname{Ai}\left(\left(\frac{x}{2}\right)^{-\frac{1}{3}}(\alpha-x)\right), \quad x \rightarrow \infty, x-\alpha=O\left(x^{1 / 3}\right) .
$$

Relation $\left(\frac{\text { as } \text { BesselAiry }}{5.3)}\right.$ was first wbtained in $[9,10]$ (see also [11, pp. 190 and 249], [12, p. 142], and bibliography within [23]). It is the principal term of series (see [24, pp. 281 and 287])

$$
J_{\alpha}(w) \approx \sum_{k=0}^{\infty}(-1)^{k}\left(\frac{x}{2}\right)^{-\frac{2 k+1}{3}}\left(P_{k}(\xi) \operatorname{Ai}(\xi)+Q_{k} \operatorname{Ai}^{\prime}(\xi)\right), \quad \xi=\left(\frac{w}{2}\right)^{-\frac{1}{3}}(\alpha-w)
$$


with polynomials $P_{k}, Q_{\text {Fig }}$ There are other relations, e.g. in [25].

Comparison on Fig. 6 shows that (5.2) gives better approximation than $(5.3)$ and can be applied on the whole semiaxis $x \in \mathbb{R}_{+}$and even for $\varepsilon \sim 1$.

\section{Acknowledgements}

Authors appreciate financial support by RFBR-CNRS project 17-51-150006.

\section{References}

[1] Landau, L. D. and Lifshitz, E. M., Theoretical Physics: Vol. 3: Quantum Mechanics: Nonrelativistic Theory, Oxford, London, Edinburgh: Pergamon Press, 1965.

[2] Lazutkin, V.F., KAM theory and semiclassical approximations to eigenfunctions, Springer, Berlin, 1993.

[3] Dobrokhotov, S. Yu., Minenkov, D.S., Shlosman, S. B., Asymptotics of wave functions of the stationary Schrödinger equation in the Weyl chamber, Theor. and Math. Phys., 2018, vol. 197, no. 2, pp. 1626-1634.

[4] Arnold, V.I., Kozlov, V.V. and Neishtadt, A.I., Mathematical Aspects of Classical and Celestial Mechanics. Dynamical Systems III. Encyclopaedia of Mathematical Sciences, 3rd ed., Springer-Verlag, New York, 2006.

[5] Dobrokhotov, S. Yu., Maslov's methods in linearized theory of gravitational waves on the liquid surface, Sov. Phys. - Dokl., 1983, vol. 28, pp. 229-231.

[6] Exner, P. and Kovařík, H., Quantum Waveguides, Springer International Publishing Switzerland, 2015.

[7] Brekhovskikh, L. M., Godin, O.A., Acoustics of Layered Media II. Point Sources and Bounded Beams, Springer-Verlag Berlin Heidelberg GmbH, 1992.

[8] Belov, V. V., Dobrokhotov, S. Yu., and Tudorovskiy, T. Ya., Operator separation of variables for adiabatic problems in quantum and wave mechanics, Journal of Engineering Mathematics, 2006, vol. 55, pp. 183-237.

[9] Wirtinger, Berichte des natur.-med. Vereins in Innsbruck, 1897, vol. XXIII, pp. 7-15.

[10] Nicholson, J. W., On the Relation of Airy's Integral to the Bessel Functions, Phil. Mag., 1909, vol. 6, no. XVII, pp. 6-17.

[11] Watson, G.N., A Treatise on the Theory of Bessel Functions, 2nd ed., UK: Cambridge University Press, 1966.

[12] Magnus, W., Oberhettinger, F., Soni, R.P., Formulas and theorems for the special functions of mathematical physics, New York: Springer Inc., 1966.

[13] Zhang, J., Linear Restriction Estimates for Schrdinger Equation on Metric Cones, Communications in Partial Differential Equations, 2015, vol.40, no. 6, pp. 995-1028

[14] Cheeger, J., Taylor, M., On the diffraction of waves by conical singularities, I, Comm. Pure Appl. Math., 1982, vol. 35, no. 3, pp. 275-331;

Cheeger, J., Taylor, M., On the diffraction of waves by conical singularities, II, Comm. Pure Appl. Math., 1982, vol. 35, no. 4, pp. 487-529.

[15] Neishtadt, A., Propagation of beams through a smoothly irregular waveguides and theory of perturbations of Hamiltonian systems, Radiophysics and Quantum Electronics, 1982, vol. 25, no. 2, pp. 157-164. 
[16] Born, M. and Oppenheimer, J.R., Zur quantentheorie der molekeln, Annalen der Physik, 1927, vol. 84, pp. 457-484.

[17] Maslov, V.P., Perturbation Theory and Asymptotic Methods, Moscow: Moscow Univ. Publ., 1965; Maslov, V.P., Théorie des perturbations et méthodes asymptotiques, Paris: Dunod, Gauthier-Villars, 1972.

[18] Peierls, R.E., Quantum Theory of Solids, Oxford: The Clarendon Press, 1955.

[19] Karasev, M. V. and Maslov, V.P., Nonlinear Poisson Brackets. Geometry and Quantization, In: Transl. Math. Monographs 119. Providence, Rhode Island: AMS, 1993.

[20] Karasev, M.V. and Maslov, V.P., Asymptotic and geometric quantization, Russ. Math. Surveys, 1984, vol. 39, pp. 133-205.

[21] Berlyand, L. V. and Dobrokhotov, S. Yu., "Operator separation of variables" in problems of short-wave asymptotics for differential equations with rapidly oscillating coefficients, Sov. Phys. - Dokl., 1987, vol. 32, pp. 714-716.

[22] Slavyanov, S., Asymptotic solutions of the one-dimensional Schrödinger equation, Providence: American Mathematical Society (Translations of mathematical monographs; vol. 151), 1996.

[23] Aleksandrova, N.I., Asymptotic formulae for the Lommel and Bessel functions and their derivatives, R. Soc. open sci., 2014, vol.1, p. 140176.

[24] Schöbe, W., Eine an die Nicholsonformel anschliessende asymptotische Entwicklung für Zylinderfunktionen, Acta Math. , 1954, vol.92, pp. 265-307.

[25] Olver, F. W. J., Some new asymptotic expansions for Bessel functions of large orders, Proc. Camb. Phil. Soc., 1952, vol.48, pp.414-427. 